\title{
Esthetic Multidisciplinary Rehabilitation of Patient with Central Incisor Agenesis
}

\author{
${ }_{1}^{1}$ Vinicius I Mascarenhas, ${ }^{2}$ Thaís P Leandrin, ${ }^{3}$ Camila C Lorenzetti, ${ }^{4}$ Camila L de Castro, ${ }^{5}$ Jose RC Saad
}

\begin{abstract}
Aim: The aim of this case report was to restore the esthetic smile of a patient with agenesis of maxillary right central incisor, while discussing the most important topics in a multidisciplinary approach.
\end{abstract}

Background: Due to the absence or loss of some teeth in the anterior region, it becomes a challenge in oral rehabilitation. Restoration of function and esthetics may require a multidisciplinary treatment that can start with periodontal, orthodontic, surgical, implantology, or restorative evaluation.

Case report: A 28-year-old patient with agenesis of the maxillary right central incisor was looking to improve the esthetics of his smile. The patient had right superior lateral incisor positioned in place of the central and the canine in the position corresponding to the lateral incisor, which compromised the dental and gingival esthetics. After analyses and digital smile design (DSD), surgeries for gingival smile correction, gingivectomy (teeth 12, 14, and 15), and gingival tissue grafts (tooth 13) were made. After a period of 2 months, preparations were made on teeth 12,13 , and 14 for ceramic laminates following the initial DSD. After 1 year of cementation, maintenance and stability of gingival margin, as well as the integrity of laminate ceramic were observed.

Conclusion: In conclusion, a correct prior planning of esthetic rehabilitation is the best way to achieve excellent results to meet the needs and objectives of each patient. Often, a multidisciplinary treatment involving surgical and restorative procedures should be taken into consideration, with the appropriate choice of techniques and materials to be used.

Clinical significance: The treatment performed on the patient allowed the esthetic improvement of gingival architecture and installation of ceramic restorations with function and esthetics similar to natural teeth.

Keywords: Connective tissue, Dental agenesis, Esthetics.

How to cite this article: Mascarenhas VI, Leandrin TP, Lorenzetti CC, de Castro CL, Saad JRC. Esthetic Multidisciplinary Rehabilitation of Patient with Central Incisor Agenesis. World J Dent 2018;9(1):43-47.

\section{Source of support: Nil}

Conflict of interest: None

\footnotetext{
${ }^{1-5}$ Department of Dental Materials and Prosthodontics, School of Dentistry, São Paulo State University, Araraquara, São Paulo Brazil

Corresponding Author: Vinicius I Mascarenhas, Department of Dental Materials and Prosthodontics, School of Dentistry, São Paulo State University, Araraquara, São Paulo, Brazil, Phone: +551633016300, e-mail: vinicius_ibiapina@hotmail.com
}

\section{BACKGROUND}

In dentistry, patients have always valued esthetics while seeking to maintain a relationship between beauty and harmony. To obtain a harmonious smile, it is necessary to esthetically analyze the face, gingival margin, lip position, and tooth support. The most sought-after treatment is conservative rehabilitation since it preserves more dental structure, which has been optimized due to the evolution of ceramic, resin, and adhesive systems.

Due to the absence or loss of some teeth in the anterior region by agenesis, periodontal problem, or as a sequel of carious lesion, it is often followed by bone resorption caused by alveolar remodeling, thus becoming a challenge in oral rehabilitation. Restoration of function and esthetics may require multidisciplinary treatment that can start with periodontal, orthodontic, surgical, implantology, or restorative evaluations. The esthetic analysis is optimized by the use of digital photography, which allows careful treatment planning and good communication with the technician and ceramist showing the patient esthetic dental and facial parameters making possible the accomplishment of a suitable diagnostic waxing as well as the individualization of porcelain laminate veneers. ${ }^{1}$

Digital smile design is a multipurpose conceptual protocol that offers significant advantages: It helps in diagnosis of nonesthetic problems through oral and facial analysis, improves communication among clinical members and between them and dental technicians, and increases patient's visual perception and motivation, increasing the case success. ${ }^{2}$

Diagnostic waxing is a fundamental step to assure the predictability and success in the final esthetic result. Furthermore, it is very helpful for patients with difficulties to imagine all the possible modifications that can be performed on their smile. In many cases, it will redefine dental morphology while also adapting to each individual. The patient approval is essential in this step to avoid dissatisfaction after the preparation of prosthetic pieces. Besides, waxing is used to make a guide that with use of a bis-acrylic resin can be transferred to the patient's mouth, giving a preview of final smile that could be obtained with proposed esthetic treatment plan. ${ }^{3,4}$

Surgeries for gingival smile correction, bone grafts, connective tissue grafts, and osseointegrated implants are some of the procedures that dentists can use prior 


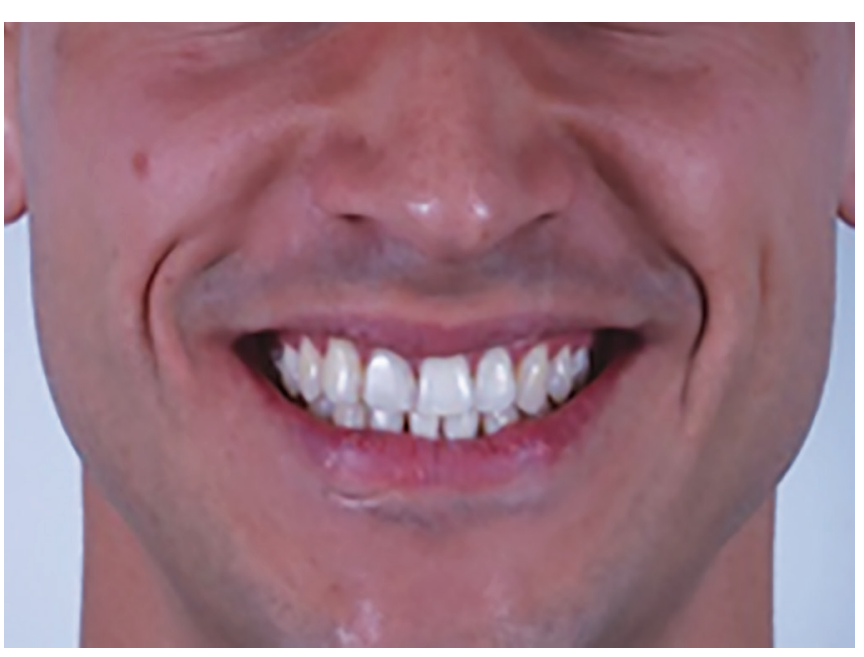

Fig. 1: Initial facial aspect

to restorative treatment. Whether for repositioning the gingival margin or for restoring tissue thickness lost over time, an esthetic/functional surgical planning should be carefully evaluated and performed. ${ }^{5}$

The demand for esthetic restorations is increasing as well as the dental ceramics use. Previously, dental ceramics were restricted only to the treatment in the anterior region, but presently, the ceramics are one of the main alternatives of restorative materials for natural tooth recovery after great loss of tooth structure due to its favorable properties, such as compressive resistance strength, thermal conductivity, similarity to dental tissues, radiopacity, marginal integrity, color stability, biomimetism, etc. Dental ceramics have great color stability, excellent optical characteristics, and sometimes have significant improvements in their composition, which allow this material to be present in high-stress mechanical situations, especially when adhered to dental structure by adhesive systems and resin cements. The ceramic laminated veneers are thin indirect restorations with indication for teeth with nonesthetic shape being able to recover their natural shape and in some cases to recover its natural color. ${ }^{6}$

\section{CASE REPORT}

A 28-year-old patient was treated at the specialization clinic of the teaching and research in dentistry Araraquarense foundation (FAEPO), seeking to improve the esthetics of his smile. It was observed that the patient presented with absence of the right central incisor (Fig. 1), and he reported the use of orthodontic treatment for long years moving the entire right arch to mesial, due to the agenesis of the central incisor. The right superior lateral incisor was positioned in place of the central and the canine in the position corresponding to the lateral incisor, which compromised the esthetic of his smile (Fig. 2).

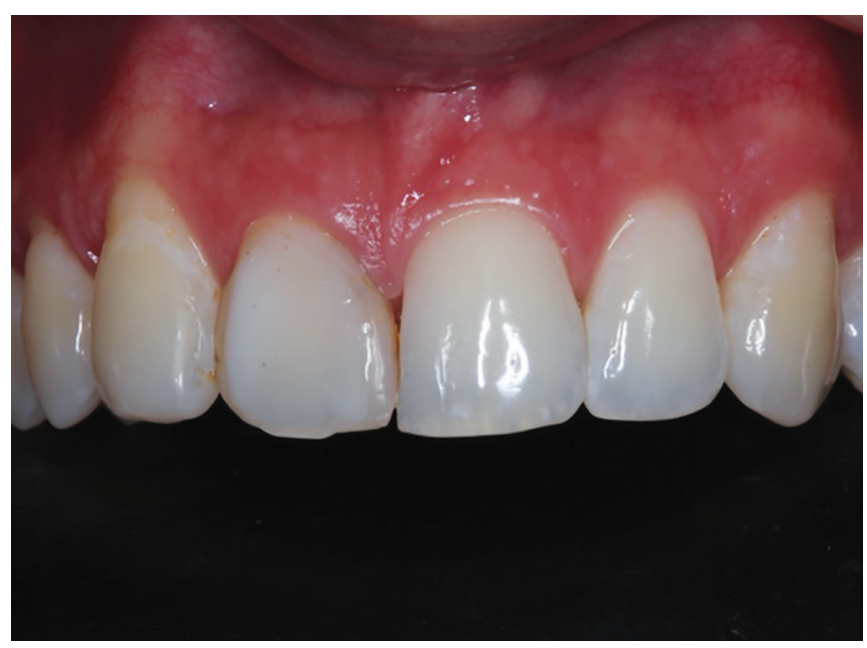

Fig. 2: Initial oral aspect

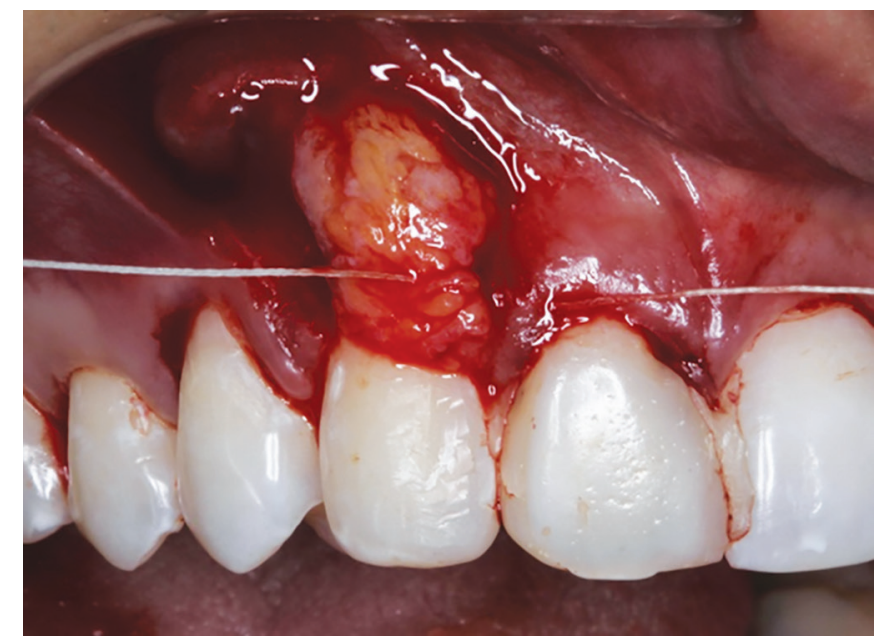

Fig. 3: Gingivectomy and connective tissue graft

After clinical and radiographic analysis and after knowing the patient expectations and interests, photos were taken to proceed to DSD to assist the treatment plan. After the digital planning, upper and lower arch impressions were obtained, making it possible to use the models to proceed with waxing based on treatment planning. A silicone guide was then made using the waxing, and a mock-up was performed on the patient using bis-acrylic resin to test the shape of the teeth, contacts, and phonetics.

To improve the harmony and esthetic of gingival architecture, it was suggested to perform a gingivectomy on the teeth 12, 14, and 15 associated with connective tissue graft, radicular recontouring, and coronal flap advanced on tooth 13 (Fig. 3).

The first clinical step of his esthetic rehabilitation treatment was the surgical procedure of gingivectomy on teeth 12,14 , and 15 using a 15C scalpel blade making an internal incision by removing 1, 1, and $2 \mathrm{~mm}$ gingiva respectively. Vertical incisions were performed on tooth 13 showing dental root and making possible to wear 


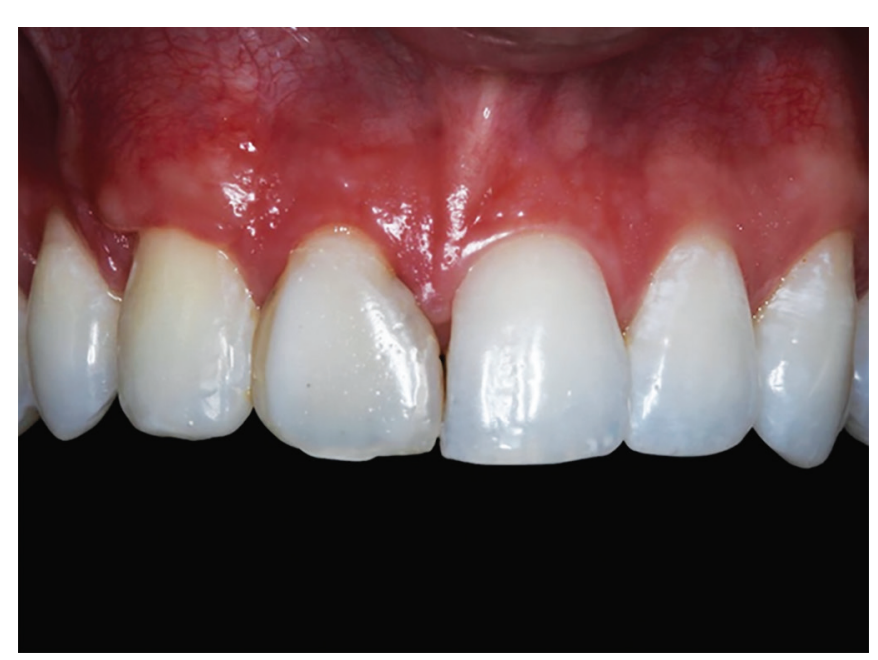

Fig. 4: Sixty days postoperative

teeth root in view of great root volume, seeking a better esthetic and prognosis of connective tissue graft (Fig. 3).

Subepithelial connective tissue graft was removed from cleft palate between teeth 23 and 26. For removal of the graft, a single 15C surgical blade was used to better control the thickness of tissue removed. After removing, the tissue was regularized with a scalpel, removing the epithelial tissue and part of the fat tissue in the largest volume. The graft tissue was then sutured on sides with 5-0 nylon and 5-0 polyglactin suture and the sutures attached to a resin applied to the buccal surface of the tooth (Fig. 3). At the end, the graft donor site was sutured with 4-0 silk thread keeping the clot.

After a period of 15 days, the sutures were removed, and after waiting for a period of 60 days (Fig. 4) esthetic restorative procedures were initiated. After this period, the teeth 12,13 , and 14 were prepared with wear on the vestibular and proximal surfaces to gain space for restorative feldspathic ceramics using 2135 fine-grained dental diamond burs (Fig. 5). After the preparations made at the gingival level, a retractor wire was placed on each tooth allowing correct impression with silicone in light and heavy consistency on both the upper and lower arches. The waxing was used to make a silicone guide that served as a template for making temporary restorations with bis-acrylic resin.

After the preparation of the ceramic veneers by ceramist, the correct adaptation was verified, proceeding with the color test using try-in paste. For cementation, the preparations were cleaned with brush and prophylactic paste, the pieces were prepared with $10 \%$ hydrofluoric acid for 1 minute and 30 seconds and then with silane. The teeth were conditioned with $37 \%$ phosphoric acid for 15 seconds in dentin and 30 seconds in enamel, washed for 45 seconds, followed by primer and bonding with adhesive system (Scotchbond; 3M ESPE, Seefeld, Germany) and polymerized for 20 seconds. The cement

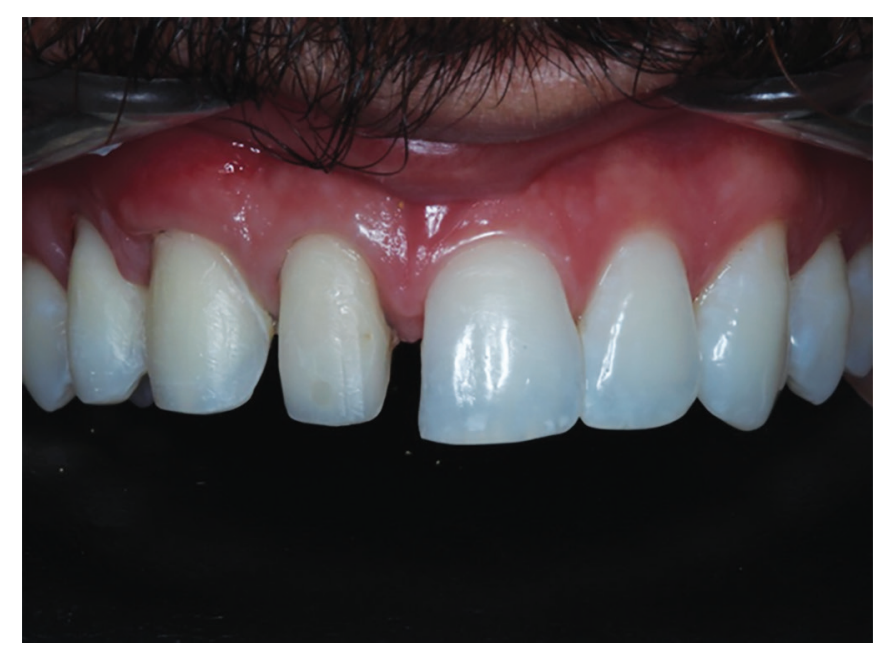

Fig. 5: Teeth preparation

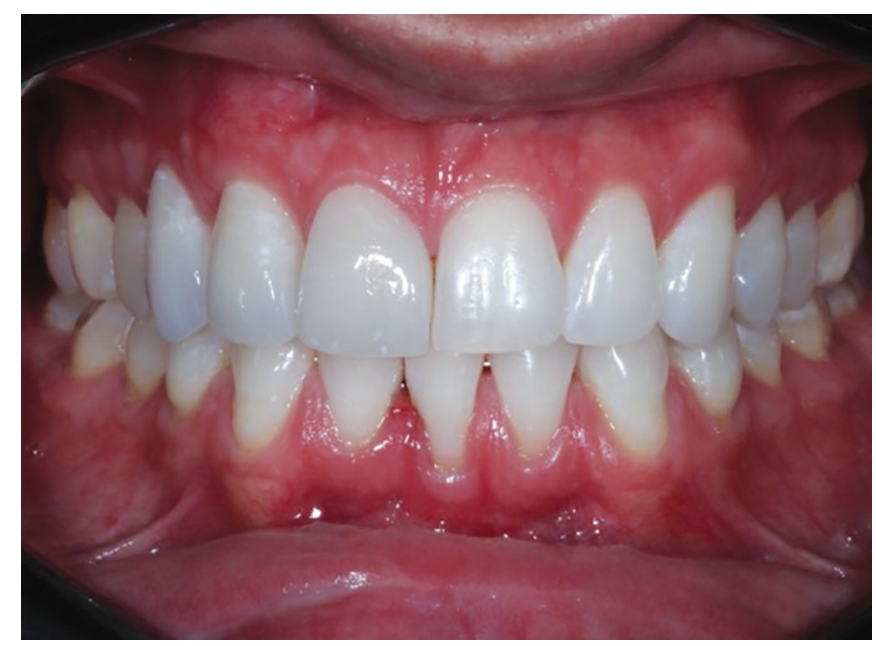

Fig. 6: One year after ceramic placement

used was the light-cured resin cement (Variolink; Ivoclar Vivadent, Schaan, Liechtenstein) translucent color, followed by removal of excess and polymerization for 40 seconds vestibular and another 40 seconds for incisal.

After 1 year, the patient returned to control case presenting stability of the results (Fig. 6).

\section{DISCUSSION}

The intense search for esthetics and harmonious smile has contributed to increase patients' expectation, consequently contributing to the search for the creation of new materials and techniques in view to performing more conservative procedures and increasingly esthetic results.

Esthetics are a highly subjective concept associated with social, cultural, and psychological factors that change according to the time, values, and age of each individual. Loss of teeth in the anterior region associated with other problems, such as no adapted crowns, restorations without adequate anatomy, or even restorative materials with pigmentation is a factor that directly influences people's self-esteem beyond masticatory function. ${ }^{7,8}$ 
The golden ratio can be used to aid in the dimensioning of teeth and smile in a frontal view where according to the literature, each tooth should be 0.618 times smaller than its adjacent more mesial one in the construction of a harmonic smile. The proportioning technique based on the golden ratio can be an excellent guide to achieve clinical esthetic success and is useful in cosmetic dentistry since it is used as an aid and adapted to facilitate the frontal analysis of teeth and smile. Thus, a pleasant smile also depends on a good proportionality analysis, not only width and height of teeth but also how these teeth are related to the lips and gums in the composition of smile. ${ }^{9}$

The concept of DSD is based on facial and dental proportion analysis using a predetermined series of highquality digital patient photos and videos to observe the relationship between teeth, gums, lips, and smile with the facial movements. Digital drawings are easily made on the images based on what is desired in terms of gain and esthetic proportion following a didactic sequence using a digital ruler to communicate precisely with the dental technician, allowing greater fidelity to the treatment planning and smile conception. This results in a clear, attractive, and understandable presentation for patients; furthermore, all communication data can be stored in a software to facilitate access for any team member to add important clinical considerations. ${ }^{10}$

In many rehabilitation treatments that require an esthetic approach involving anterior region of smile, it is necessary to plan using diagnostic waxing of the cases, making possible to perform a mockup with bis-acrylic resin and definitive ceramic pieces following the same anatomy proportion. ${ }^{10}$

Gingival recessions can arise from a variety of causes (tooth positioning in arch, thin gingival biotype, trauma, and orthodontic movement); moreover, cosmetic problems can be more susceptible to acid, traumatic brushing, and caries, which increase the risk of abrasions and erosion, because of which the root covering either by surgical or restorative means is absolutely necessary. ${ }^{11}$ The treatment of gingival recessions by surgical means can occur through several techniques, and one of the more used and that has been reported with excellent results in the literature is the coronal flap advancement associated with subepithelial connective tissue graft. ${ }^{12}$ The connective tissue graft allows the gain of gingival thickness and marginal stability, ensuring better results in Miller's class I and II recessions. ${ }^{13,14}$

A systematic review in $2012^{15}$ correlated papers that evaluated the predictability of root coverage in Miller's class I, II, and III recessions. The authors concluded that root coverage with coronal flap advancement is a technique with good predictability results presenting an average coverage of 91.5 to $97.27 \%$ in Miller's class I and II recessions. The authors also concluded that association of connective tissue grafts with advanced coronal flap promotes a greater percentage of complete root coverage ( $35 \%$ of the flap, $52 \%$ of the flap + connective tissue graft) and long-term stability. ${ }^{15}$

Known as Langer and Langer ${ }^{16}$ technique, the use of vertical incisions was chosen in this case because it allows greater advanced flap besides allowing the reduction of canine root volume seeking to improve esthetics and give more stability to gingival margin of tooth 13 .

The feldspathic ceramics have excellent optical properties, good color stability, and excellent adhesion to tooth preparation when using the correct cementing agent. Ceramic restorations can be produced by several different techniques and materials presenting specific characteristics. Bagis and Turgut ${ }^{17}$ evaluated the optical properties, such as chroma and translucency of different types of ceramics (e.max Press, e.max CAD, Empress Esthetic, e.max Ceram, Inline, and ZirPress systems). One of the authors' findings showed that even the feldspathic ceramics that present some components to improve mechanical characteristics, such as leucite (Empress Esthetic) show higher translucency than the lithium disilicate-based ceramics made in single block for computer-aided design/computer-aided manufacturing milling (e.max CAD). This is one of the characteristics that make feldspathic ceramics a good material of choice for anterior esthetic restorations of teeth with little color change. ${ }^{17}$

\section{CONCLUSION}

A correct prior planning of esthetic rehabilitation is the best way to achieve excellent results according to patients' needs. Often, a multidisciplinary treatment involving surgical and restorative procedures should be taken into consideration with appropriate choice of techniques and materials used. In addition, the patients who present with agenesis or the absence of teeth in the anterior region should have a more careful orthodontic treatment plan to avoid bone and root volume interfering with esthetic smile.

\section{CLINICAL SIGNIFICANCE}

The treatment performed on patient allowed the esthetic improvement of gingival architecture and installation of ceramic restorations with function and esthetics similar to those of natural teeth.

\section{REFERENCES}

1. Belser UC, Magne P, Magne M. Ceramic laminate veneers: continuous evolution of indications. J Esthet Dent 1997 Jul;9(4):197-207. 
2. Coachman C, Paravina RD. Digitally enhanced esthetic dentistry-from treatment planning to quality control. J Esthet Restor Dent 2016 Mar;28(Suppl 1):S3-S4.

3. Behle C. Placement of direct composite veneers utilizing a silicone buildup guide and intraoral mock-up. Pract Period Aesthet Dent 2000 Apr;12(3):259-266.

4. Fradeani M, Barducci G, Bacherini L. Esthetic rehabilitation of a worn dentition with a minimally invasive prosthetic procedure (MIPP). Int J Esthet Dent 2016 Spring;11(1):16-35.

5. Force J, Niemiec B. Gingivectomy and gingivoplasty for gingival enlargement. J Vet Dent 2009 Summer;26(2):132-137.

6. Alhekeir DF, Al-Sarhan RA, Al Mashaan AF. Porcelain laminate veneers: clinical survey for evaluation of failure. Saudi Dent J 2014 Apr;26(2):63-67.

7. Soares PV, Spini PH, Carvalho VF, Souza PG, Gonzaga RC, Tolentino AB, Machado AC. Esthetic rehabilitation with laminated ceramic veneers reinforced by lithium disilicate. Quintessence Int 2014 Feb;45(2):129-133.

8. Jordan A. Clinical aspects of porcelain laminate veneers: considerations in treatment planning and preparation design. J Calif Dent Assoc 2015 Apr;43(4):199-202.

9. Mahshid M, Khoshvaghti A, Varshosaz M, Vallaei N. Evaluation of "golden proportion" in individuals with an esthetic smile. J Esthet Restor Dent 2004 May;16(3):185-192.

10. Manjula WS, Sukumar MR, Kishorekumar S, Gnanashanmugam K, Mahalakshmi K. Smile: a review. J Pharm Bioallied Sci 2015 Apr;7(Suppl 1):S271-S275.
11. Chambrone L, Tatakis DN. Periodontal soft tissue root coverage procedures: a systematic review from the AAP regeneration workshop. J Periodontol 2015 Feb;86(Suppl 2): S8-S51.

12. Zucchelli G, Amore C, Sforza NM, Montebugnoli L, De Sanctis M. Bilaminar techniques for the treatment of recession-type defects. A comparative clinical study. J Clin Periodontol 2003 Oct;30(10):862-870.

13. Miller PD Jr. A classification of marginal tissue recession. Int J Periodontics Restorative Dent 1985;5(2):8-13.

14. Azaripour A, Kissinger M, Farina VS, Van Noorden CJ, Gerhold-Ay A, Willershausen B, Cortellini P. Root coverage with connective tissue graft associated with coronally advanced flap or tunnel technique: a randomized, doubleblind, mono-centre clinical trial. J Clin Periodontol 2016 Dec;43(12):1142-1150.

15. Hofmanner P, Alessandri R, Laugisch O, Aroca S, Salvi GE, Stavropoulos A, Sculean A. Predictability of surgical techniques used for coverage of multiple adjacent gingival recessionsa systematic review. Quintessence Int 2012 Jul-Aug;43(7): 545-554.

16. Langer B, Langer L. Subepithelial connective tissue graft technique for root coverage. J Periodontol 1985 Dec;56(12): 715-720.

17. Bagis B, Turgut S. Optical properties of current ceramics systems for laminate veneers. J Dent 2013 Aug;41(Suppl 3): e24-e30. 